\title{
EHP SPECTRA AND PERIODICITY. I: GEOMETRIC CONSTRUCTIONS
}

\author{
BRAYTON GRAY
}

\begin{abstract}
The techniques used in EHP calculation are studied, and lead to the notion of an EHP spectrum. A simple inductive procedure suggests the existence of higher order EHP spectra in which the first differential corresponds to $v_{n}$ multiplication. The next case $(n=1)$ is constructed using the work of Cohen, Moore, and Neisendorfer. Some of the expected universal properties are proven.
\end{abstract}

It is the purpose of this note to show how some very elementary considerations about how the EHP spectral sequence works can be generalized and combined with some constructions of Cohen, Moore, and Neisendorfer [CMN $1,2,3]$. If these constructions can be carried out in general it would yield a new unstable development for the spectra $V(m)$ of Smith and Toda [S, T3]. Thus we add to the problem of constructing the spectra $V(m)$, the problem of constructing an unstable filtration together with EHP sequences generalizing the classical EHP sequences when $m=-1$. If this approximation to $V(m)$ can be constructed, we shall see that $V(m+1)$ exists. Thus, among other things, we present a new inductive approach to constructing $V(m)$. In particular, sparseness arguments do not appear.

The first new case is $m=0$ and we will discuss this at some length. The results of Cohen, Moore, and Neisendorfer and some recent work of Anick are germane to the construction. We also indicate an inductive approach to the general problem, which we intend to pursue.

I wish to thank Mark Mahowald, who first alerted me to the notion of higher order suspension, and whose intuition has been a valuable source. Numerous conversations with Rob Thompson have been valuable throughout this work.

There are a number of systematic ways of working with the EHP spectral sequence. One of the simplest and most fruitful is with the use of the BarrattToda formula [B1, T1]. Suppose $\alpha \in \pi_{k}\left(S^{n}\right)$ and $\beta \in \pi_{l}\left(S^{m}\right)$. Then by the Barratt-Hilton formula [BH] the composites $\alpha \beta$ and $\beta \alpha$ are equal on $S^{n+m}$, i.e., $E^{m} \alpha \circ E^{k} \beta= \pm E^{n} \beta \circ E^{l} \alpha$ as elements of $\pi_{k+l}\left(S^{m+n}\right)$. Let us write $[\alpha, \beta]=$ $E^{m-1} \alpha \circ E^{k-1} \beta \mp E^{n-1} \beta \circ E^{l-1} \alpha$, so $E[\alpha, \beta]=0$. (Note: this is a commutator, not a Whitehead product!) Now from the EHP sequences, $[\alpha, \beta]$ must be in

Received by the editors May 6, 1991.

1980 Mathematics Subject Classification (1985 Revision). Primary 55P99, 55Q52. 
the image of $P$. If either $\alpha$ or $\beta$ is a suspension, $[\alpha, \beta]=0$ so $[\alpha, \beta]$ must depend only on $H(\alpha)$ and $H(\beta)$. In fact

Theorem 1.1 (Barratt-Toda) [B1, T1]. Localized at 2,

$$
[\alpha, \beta]=P(E(H(\alpha) \wedge H(\beta))) .
$$

This is effective in calculation since $P$ determines the differential. Throughout the calculation one makes a list of all possible composites. This is aided by the fact that under mild hypothesis ${ }^{1} H(\alpha \circ \beta)=H(\alpha) \circ \beta$. One then follows the composites $\alpha \circ \beta$ and $\beta \circ \alpha$ and finds that the stable relation $\alpha \beta= \pm \beta \alpha$ is enforced by the relevant differential on $E(H(\alpha) \wedge H(\beta))$ which itself is a composite $H(\alpha) \circ H(\beta)$ or $H(\beta) \circ H(\alpha)$.

Localized at an odd prime a similar situation occurs. We need to take account, in this case, for the fact that $E: \pi_{r}\left(S^{2 s-1}\right) \rightarrow \pi_{r+1}\left(S^{2 s}\right)$ is a monomorphism. Thus if $m$ and $n$ are odd, $[\alpha, \beta]=0$ and we construct a commutator on $S^{m+n-2}$. It lies in the bracket $\{H(\alpha), p l, H(\beta)\}$.

Now we enquire whether there are other spectra which could enjoy these properties. We ask for a spectrum $X=\left\{X^{n}\right\}$ together with fibrations:

$$
\ldots \stackrel{P}{\longrightarrow} X^{n-1} \stackrel{E}{\longrightarrow} \Omega X^{n} \stackrel{H}{\longrightarrow} \Omega X^{f(n)-1}
$$

which we will refer to as EHP fibrations. We would like a "Barratt-Toda formula" to hold. We need, then, to define "compositions" in the homotopy of $X$. Given a "unit" $u_{l}: S^{l} \rightarrow X^{l}$ and a homotopy class $\beta: S^{l} \rightarrow X^{m}$ we then seek an extension:

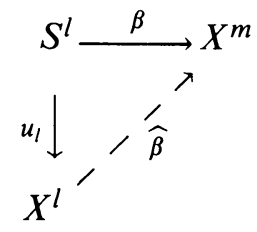

possibly with some uniqueness property. In this case we can define compositions, by $\beta$ o $\alpha=\widehat{\beta} \circ \alpha$ for $\alpha \in \pi_{*}\left(X^{l}\right)$. In favorable circumstances this will induce a bilinear multiplication in $\pi_{*}(X)$. Now let us assume that $X$ is a ring spectrum with unit $u$ and multiplication $\mu: X^{n} \wedge X^{n} \rightarrow X^{n+m}$ which is homotopy associative (compositions are associative) and homotopy commutative (the Barratt-Toda formula will enforce this). Suppose that among the uniqueness properties for $\widehat{\beta}$ we require that it be an $X$ module map; i.e., we have a commutative diagram

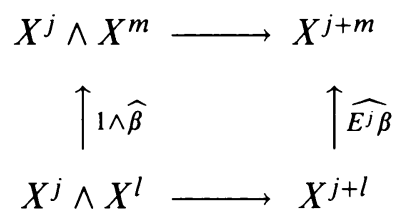

This guarantees that $\pm E^{n} \beta \widehat{o} E^{l} \alpha=\mu(\alpha \wedge \beta)= \pm E^{m} \alpha \hat{\circ} E^{k} \beta$. Now given $\alpha \in \pi_{k}\left(X^{n}\right)$ and $\beta \in \pi_{l}\left(X^{m}\right)$, we can define a commutator $[\alpha, \beta] \in$

\footnotetext{
${ }^{1}$ Localized at an odd prime we conjecture that no hypothesis is needed ([G3; Theorem 1] implies half of the cases).
} 
$\pi_{k+l-1}\left(X^{n+m-1}\right)$ as before. Furthermore $E[\alpha, \beta] \sim *$, and so $[\alpha, \beta]=P(\gamma)$ where $\gamma: S^{k+l+1} \rightarrow X^{f(n+m)-1}$. Now consider the composition:

$$
S^{k} \wedge S^{l} \stackrel{H(\alpha) \wedge H(\beta)}{\longrightarrow} X^{f(n)-1} \wedge X^{f(m)-1} \stackrel{\mu}{\longrightarrow} X^{f(n)+f(m)-2} .
$$

If a Barratt-Toda formula were to hold we would need $f(n)+f(m)=f(n+m)$. This implies that $f(n)=k n$ where $k=f(1)$.

Let us now confine our attention to odd primes where we find our examples. Using the sphere spectrum as a model, we can only expect the usual BarrattToda formula to hold when one of $n$ and $m$ is even. Thus $f(2 n)=h n$ and $f(2 n+1)=h n+f(1)$. Both because of some technicalities that will soon come up, and because of the examples we have in mind, we will assume that both $h$ and $f(1)$ are even.

Definition 1.2. An EHP spectrum of period $2 d$ is a $p$-local spectrum $X=$ $\left\{X^{n}\right\}_{n \geq 0}$ together with fibrations:

$$
\begin{aligned}
& \ldots \stackrel{P}{\longrightarrow} X^{2 n-1} \stackrel{E}{\longrightarrow} \Omega X^{2 n} \stackrel{H^{\prime}}{\longrightarrow} \Omega X^{2 k n-1}, \\
& \ldots \stackrel{P}{\longrightarrow} X^{2 n} \stackrel{E}{\longrightarrow} \Omega X^{2 n+1} \stackrel{H}{\longrightarrow} \Omega X^{2 k n+2 d+1} .
\end{aligned}
$$

Here $h=2 k$ and $f(1)=2 d+2$. It is important to observe that this is an unstable property of a spectrum. Two spectra $X$ and $Y$ could be stably homotopy equivalent while only one is an EHP spectrum. The spaces $X^{n}$ thus give a favorable unstable development of a stable homotopy type. We do not require either that $X$ be connective or a ring spectrum. In fact, if $X$ is an EHP spectrum, $\Omega X=\left\{\Omega X^{n}\right\}_{n \geq 0}$ is also an EHP spectrum stably equivalent to $\Sigma^{-1} X$.

We shall see that in the construction process that follows, it is often easier to construct $\Omega^{s} X^{k}$ than to construct $X^{k}$ for some $s>0$. This is inconsequential for a homotopy spectral sequence. All of the examples of interest have $k=p$, and $k=p^{i}$ seems almost necessary if the construction of $H$ is natural enough. The cases of interest, if they can be delooped to be ring spectra, will have $X^{0} \simeq \mathbf{Z}_{p}$ with the discrete topology.

The composite $\Delta_{n}=H^{\prime} \circ \Omega P$ :

$$
\Omega^{3} X^{2 n k+2 d+1} \longrightarrow \Omega X^{2 n} \longrightarrow \Omega X^{2 n k-1}
$$

will be called the periodicity operator. In favorable cases it corresponds to a self-map of $X$ of degree $2 d$.

In the case of the sphere spectrum, $\Delta_{n}=\Omega \varphi_{n}$ for some map $\varphi_{n}: \Omega^{2} S^{2 n p+1} \rightarrow$ $S^{2 n p-1}$ [G4]. Maps of this type have been constructed by Cohen, Moore, and Neisendorfer [CMN1, CMN2]. They have constructed a sequence of maps $\pi_{n}: \Omega^{2} S^{2 n+1} \rightarrow S^{2 n-1}$ such that the diagrams

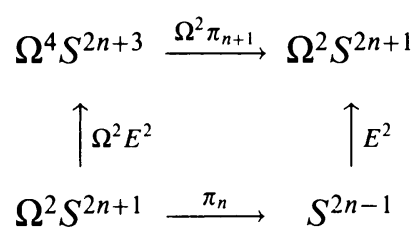

homotopy commute for each $n \geq 1$. We formulate this as 
Property $C M N$. There are maps $\pi_{n}: \Omega^{2} X^{2 n+2 d+1} \rightarrow X^{2 n-1}$ for $n \geq 1$ such that the diagrams:

$$
\Omega^{4} X^{2 n+2 d+3} \stackrel{\Omega^{2} \pi_{n+1}}{\longrightarrow} \Omega^{2} X^{2 n+1}
$$

commute and $\Delta_{n} \sim \Omega \pi_{n k}$.

$$
\begin{array}{ccr}
\uparrow \Omega^{2} E^{2} & & \uparrow E^{2} \\
\Omega^{2} X^{2 n+2 d+1} \stackrel{\pi_{n}}{\longrightarrow} X^{2 n-1}
\end{array}
$$

The significance of this property is illuminated by the following.

Theorem 1.3. Suppose that $X$ is an EHP spectrum of period $2 d$ satisfying $C M N$. Then there is a derived EHP spectrum $Y$ of period $2 d^{\prime}$ where $d^{\prime}=(d+1) k-1$. Proof. We define spaces $Y^{n}$ as the fibers in the following fibrations:

$$
\begin{aligned}
& Y^{2 n-1} \longrightarrow \Omega^{2} X^{2 n+2 d+1} \stackrel{\pi_{n}}{\longrightarrow} X^{2 n-1}, \\
& Y^{2 n} \longrightarrow \Omega X^{2 n+2 d+1} \stackrel{\Omega \rho_{n+1}}{\longrightarrow} \Omega X^{2 n+1},
\end{aligned}
$$

where $\rho_{n+1}=\pi_{n+1} \circ E^{2}$. Now let $W_{n} \rightarrow X^{2 n-1} \stackrel{E^{2}}{\longrightarrow} \Omega^{2} X^{2 n+1}$ be a fibration; $W_{n}$ is also the fiber of $\Delta_{n}$ as usual. This follows from the diagram:

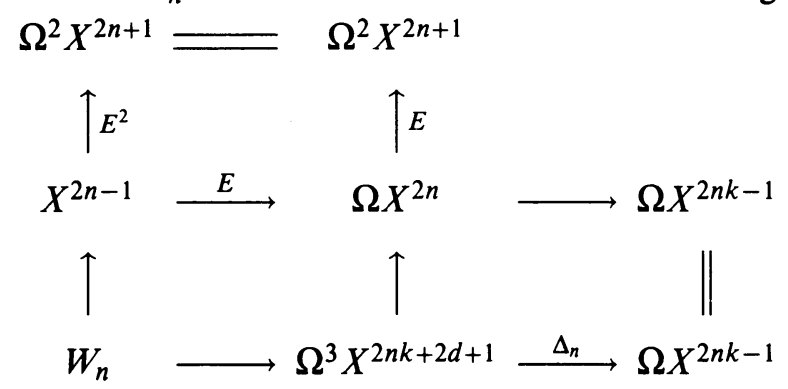

so $W_{n} \simeq \Omega Y^{2 n k-1}$. We now construct the EHP fibrations for $Y$ with the following diagrams of fibrations:

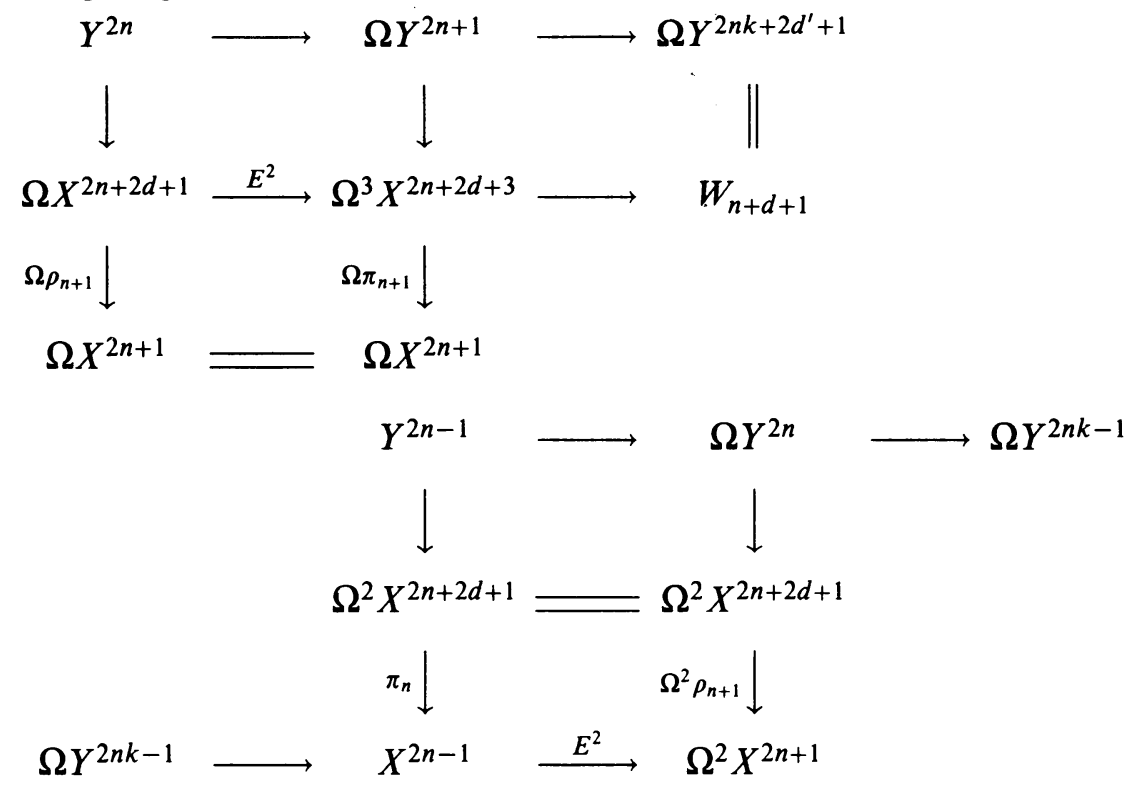

This completes the proof. 
Let us assume that for suitable primes $p$, this process can be iterated $m+1$ times beginning with the sphere spectrum. This would require that we prove CMN at each stage in order to go on. We will first look at the sequence of periods. Beginning with $d=0$ and using the formula $d^{\prime}=(d+1) p-1$, we obtain the sequence $q_{0}, q_{1}, \ldots, q_{m+1}$ where $q_{m}=2\left(p^{m}-1\right)$. As we shall see, the maps $\pi_{n}$ and $\rho_{n}$ are compressions of internal $v_{m}$ self-maps. Let us now display the fibration sequence that we would get in this case. Write $W_{(m)}^{n}$ for the $n$th space we would get in the $(m+1)$ th $k$ iteration. Thus $W_{(-1)}^{n}=S^{n}$ if $n$ is odd and $J_{p-1}\left(S^{n}\right)$ if $n$ is even. ${ }^{2}$ As we shall see, the space $\left\{W_{(m)}^{n}, n \geq 0\right\}$ form a spectrum equivalent to $\Sigma^{-m-1} V(m)$, where $V(m)$ is the Smith-Toda complex [Sm, T2]. We would then get two EHP sequences:

$$
\begin{aligned}
& H: \ldots \stackrel{P}{\longrightarrow} W_{(m)}^{2 n} \stackrel{E}{\longrightarrow} \Omega W_{(m)}^{2 n+1} \stackrel{H}{\longrightarrow} \Omega W_{(m)}^{2 n p+q_{m}+1}, \\
& H^{\prime}: \ldots \stackrel{P}{\longrightarrow} W_{(m)}^{2 n-1} \stackrel{E}{\longrightarrow} \Omega W_{(m)}^{2 n} \stackrel{H^{\prime}}{\longrightarrow} \Omega W_{(m)}^{2 n p-1} .
\end{aligned}
$$

There would be a sequence corresponding to the double suspension:

$$
E^{2}: \ldots \rightarrow W_{(m)}^{2 n-1} \stackrel{E^{2}}{\longrightarrow} \Omega^{2} W_{(m)}^{2 n+1} \rightarrow W_{(m+1)}^{2 n p-1}
$$

Finally there would be the defining Cohen-Moore-Neisendorfer sequence and a restricted version:

$$
\begin{aligned}
& C M N: \ldots \rightarrow \Omega W_{(m-1)}^{2 n-1} \rightarrow W_{(m)}^{2 n-1} \rightarrow \Omega^{2} W_{(m-1)}^{2 n+q_{m}+1} \stackrel{\pi_{n}}{\longrightarrow} W_{(m-1)}^{2 n-1} \\
& R C M N: \ldots \rightarrow \Omega^{2} W_{(m-1)}^{2 n+1} \rightarrow W_{(m)}^{2 n} \rightarrow \Omega W_{(m-1)}^{2 n+q_{m}+1} \stackrel{\Omega \rho_{n+1}}{\longrightarrow} \Omega W_{(m-1)}^{2 n+1}
\end{aligned}
$$

A noteworthy consequence is the following:

Proposition 1.4. If there is an EHP spectrum $\left\{W_{(m)}^{n}\right\}$ representing $\Sigma^{-m-1} V(m)$ as above, the spectrum $V(m+1)$ exists; furthermore if $\left\{W_{(m)}^{n}\right\}$ satisfies condition $C M N,\left\{W_{(m+1)}^{n}\right\}$ represents $\Sigma^{-m-2} V(m+1)$.

Proof. Since $W_{(m)}^{n}$ is $n-m-2$ connected, $E: W_{(m)}^{2 n} \rightarrow \Omega W_{(m)}^{2 n+1}$ is a $2 n p+q_{m}-$ $m-2$ isomorphism and $E: W_{(m)}^{2 n-1} \rightarrow \Omega W_{(m)}^{2 n}$ is a $2 n p-m-4$ isomorphism; whence $E: W_{(m)}^{2 n-1} \rightarrow \Omega^{2 r} W_{(m)}^{2 n+2 r-1}$ is a $2 n p-m-4$ isomorphism. Thus if $n$ is large $W_{(m)}^{2 n-1}=\left(\Sigma^{2 n-m-2} V(m)\right) \cup$ higher cells. In particular, for $n$ large, we have a factorization:

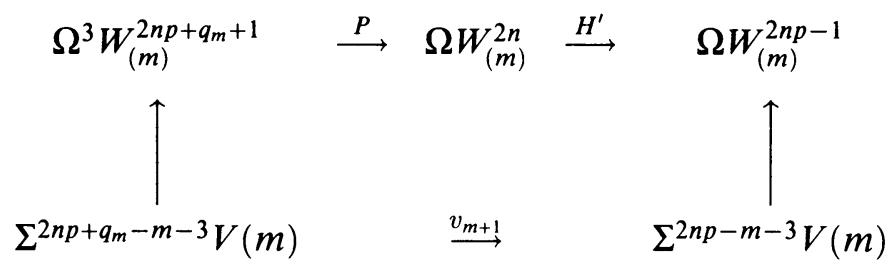

Let $\Sigma^{2 n p-m-3} V(m+1)$ be the mapping cone. By an argument in $\S 5, Q^{m+1} \neq 0$ in $V(m+1)$ so it is the desired space.

\footnotetext{
${ }^{2}$ Throughout this work we will write $\widehat{S}^{2 n}$ for $J_{p-1}\left(S^{2 n}\right)$.
} 
In [G7] we show that there are $\Lambda$ algebra models for each of these spaces, and short exact sequences for each of these fibrations. In particular, there is an EHP spectral sequence for $\operatorname{Ext}_{A_{p}}\left(H^{*}(V(m))\right)$ generalizing the Bousfield-Curtis spectral sequence when $m=-1$.

From the sequence $C M N$ we see that there is a map $\Omega W_{(m-1)}^{n} \rightarrow W_{(m)}^{n}$. By iteration we get a map $\Omega^{m+1} S^{n} \rightarrow W_{(m)}^{n}$ which is an $n-m-1$ isomorphism, and by 1.4, $\left\{W_{(m)}^{n}\right\} \simeq \Sigma^{-m-1} V(m)$. Our original analysis required a ring spectrum with unit, so this suggests that we might try to write $\left\{W_{(m)}^{n}\right\}=\Omega^{m+1}\left\{V_{m}^{n}\right\}$ delooping the spaces and maps $m+1$ times. This possibility is encouraged by [G7, Proposition 7.5]. As we shall see, this in fact happens when $m=0$. In the inductive construction of the derived EHP spectrum $Y$ each $Y^{2 n}$ was given as a loop space; in fact we have a fibration

$$
Y^{2 n} \rightarrow \Omega X^{2 n+2 d+1} \rightarrow \Omega X^{2 n+1} \rightarrow B Y^{2 n} \rightarrow X^{2 n+2 d+1} \stackrel{\rho_{n+1}}{\rightarrow} X^{2 n+1} .
$$

To deloop $Y$ we also need a fibration

$$
Y^{2 n-1} \rightarrow \Omega^{2} X^{2 n+2 d+1} \stackrel{\pi_{n}}{\longrightarrow} X^{2 n-1} \rightarrow B Y^{2 n-1} \stackrel{\gamma_{n}}{\longrightarrow} \Omega X^{2 n+2 d+1}
$$

and a lifting of $\gamma_{n}$ to $Y^{2 n}$. Let us examine these fibrations in case $m=0$, i.e. $X^{2 n+1}=S^{2 n+1}$ and $d=0$. The first is

$$
Y^{2 n} \longrightarrow \Omega S^{2 n+1} \stackrel{p}{\longrightarrow} \Omega S^{2 n+1} \longrightarrow B Y^{2 n} \longrightarrow S^{2 n+1} \stackrel{p}{\longrightarrow} S^{2 n+1} .
$$

Clearly $B Y^{2 n}=S^{2 n+1}\{p\}$. The second fibration is

$$
Y^{2 n-1} \longrightarrow \Omega^{2} S^{2 n+1} \stackrel{\pi_{n}}{\longrightarrow} S^{2 n-1} \longrightarrow B Y^{2 n-1} \stackrel{\gamma_{n}}{\longrightarrow} \Omega S^{2 n+1} .
$$

Such a fibration occurs in the work of Anick [A, Proposition 15.1]. A lift of $\gamma_{n}$ to a map $B Y^{2 n-1} \longrightarrow Y^{2 n}=\Omega S^{2 n+1}\{p\}$ is provided in [AG].

The general pattern of deloopings would then have delooped fibrations:

$$
\begin{aligned}
& B C M N: \ldots \rightarrow \Omega^{2} V_{(m-1)}^{2 n+q_{m}+1} \stackrel{\pi_{n}}{\rightarrow} V_{(m-1)}^{2 n-1} \rightarrow V_{(m)}^{2 n-1} \stackrel{\gamma_{n}}{\longrightarrow} \Omega V_{(m-1)}^{2 n+q_{m}+1}, \\
& B R C M N: \ldots \rightarrow \Omega V_{(m-1)}^{2 n+1} \rightarrow V_{(m)}^{2 n} \rightarrow V_{(m-1)}^{2 n+q_{m}+1} \stackrel{\rho_{n+1}}{\longrightarrow} V_{(m-1)}^{2 n+1} .
\end{aligned}
$$

The other sequences $E^{2}, H$, and $H^{\prime}$ would be the same as before with $V_{(m)}^{n}$ in place of $W_{(m)}^{n}$ throughout. Finally $\left\{V_{(m)}^{n}\right\}=V(m)$. This delooping is supported by [G7, Proposition 7.5].

Let us we consider the consequences of another assumption: that the map $\rho_{1}: V_{(m-1)}^{q_{m}+1} \rightarrow V_{(m-1)}^{1}$ has "Hopf invariant one"; i.e. the composite $\Omega V_{(m-1)}^{q_{m}+1} \stackrel{\Omega \rho_{1}}{\longrightarrow}$ $\Omega V_{(m-1)}^{1} \stackrel{H}{\longrightarrow} \Omega V_{(m-1)}^{q_{m}+1}$ is the identity. In this case $V_{(m)}^{0} \simeq V_{(m-1)}^{0}$ as we can see from the diagram:

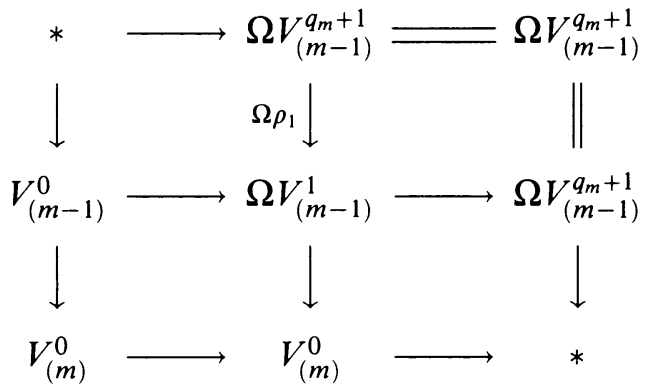


As we shall see $V_{(0)}^{0}=Z_{p}$ with the discrete topology. This suggests that $V_{(m)}^{0}=$ $\mathbf{Z}_{p}$ and this is supported by [G7, Corollary 3.6].

At this point we will discuss our main new example: $V(0)$. As we shall see it comes very close to fitting the above scheme, and does produce a derived EHP spectral sequence for the stable homotopy of $V(0)$.

The difficulty lies in property CMN. In [CMN1], the authors construct maps $\pi_{n}: \Omega^{2} S^{2 n+1} \rightarrow S^{2 n-1}$ for $n \geq 1$ such that the rectangles $(*)$ of property CMN commute, but leave open the question of whether $\Delta_{n} \sim \Omega \pi_{n p}$. In [G4 and G5] we construct a map $\varphi_{n}: \Omega^{2} S^{2 n p+1} \rightarrow S^{2 n p-1}$ with $\Delta_{n} \sim \Omega \varphi_{n}$. The reasonable procedure then is to define:

$$
\pi_{n}= \begin{cases}\pi_{n} \text { from }[\mathrm{CMN} 1] & \text { if }(n, p)=1, \\ \varphi_{m} & \text { if } n=p m\end{cases}
$$

We then need to show that the rectangles involving $\varphi_{m}$ commute. Since $\varphi_{m} \mid S^{2 m p-1}$ is the map of degree $p$, the only verification necessary is that the diagram:

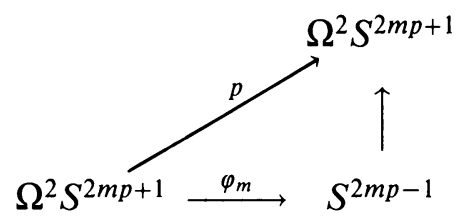

commutes. The main result of $[\mathrm{H}]$ is that the loops on this diagram:

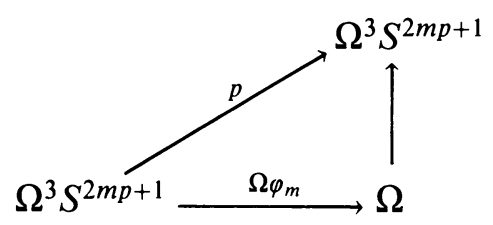

commutes. Consequently we may construct the loops on the desired EHP sequences and obtain:

Theorem 2.1. There are fibration sequences:

$$
\begin{array}{ll}
H: & \ldots \longrightarrow W_{(0)}^{2 n} \longrightarrow \Omega W_{(0)}^{2 n+1} \longrightarrow \Omega W_{(0)}^{2 n p+q+1} \\
\Omega H^{\prime}: & \ldots \longrightarrow W_{(0)}^{2 n-1} \longrightarrow \Omega^{2} W_{(0)}^{2 n} \longrightarrow \Omega^{2} W_{(0)}^{2 n p-1}, \\
C M N: & \ldots \longrightarrow W_{(0)}^{2 n-1} \longrightarrow \Omega^{2} S^{2 n+1} \stackrel{\pi_{n}}{\longrightarrow} S^{2 n-1}, \\
R C M N: & \ldots \longrightarrow W_{(0)}^{2 n} \longrightarrow S^{2 n+1} \stackrel{p}{\longrightarrow} \Omega S^{2 n+1} .
\end{array}
$$

Furthermore, if $(n, p)=1$, the fibration $\Omega H^{\prime}$ can be delooped, giving:

$$
H^{\prime}: \ldots \longrightarrow W_{(0)}^{2 n-1} \longrightarrow \Omega W_{(0)}^{2 n} \longrightarrow \Omega W_{(0)}^{2 n p-1} .
$$

Finally the spaces $\left\{W_{(0)}^{n}\right\}$ form a spectrum equivalent to $\Sigma^{-1} V(0)$.

Clearly this whole matter could be greatly simplified if we could find maps $\pi_{n p}: \Omega^{2} S^{2 n p+1} \rightarrow S^{2 n p-1}$ satisfying both (a) $p \sim E^{2} \pi_{n p}: \Omega^{2} S^{2 n p+1} \rightarrow S^{2 n p-1} \rightarrow$ 
$\Omega^{2} S^{2 n p+1}$ and (b) $\pi_{n p} \cdot H_{n} \sim *: \Omega^{2} S^{2 n+1} \rightarrow \Omega^{2} S^{2 n p+1} \rightarrow S^{2 n p-1}$. In this case we could replace $\Omega H^{\prime}$ by $H^{\prime}$ for all $n$.

In this section we will exploit some powerful results of David Anick. Relying heavily on the results in [A], the authors of [AG] construct the fibrations $B C M N$ of $\S 1$ for $V(0)$. Unfortunately we confront yet another construction for a map $\pi_{n}: \Omega^{2} S^{2 n+1} \rightarrow S^{2 n-1}$. It does, however, give us the opportunity to discuss compositions from the point of view of $\S 1$.

Theorem 3.1 [AG]. Let $p \geq 5$. Then there is a fibration sequence:

$$
\ldots \longrightarrow \Omega^{2} S^{2 n+1} \stackrel{\phi_{n}}{\longrightarrow} S^{2 n-1} \longrightarrow T^{2 n-1} \stackrel{\gamma_{n}}{\longrightarrow} \Omega S^{2 n+1}
$$

where $T^{2 n-1}$ is an $H$ space, $\gamma_{n}$ is an $H$ map, $\phi_{n}$ has degree $p$ and $\Omega^{2} S^{2 n+1}$ $\stackrel{\phi_{n}}{\longrightarrow} S^{2 n-1} \stackrel{E^{2}}{\longrightarrow} \Omega^{2} S^{2 n+1}$ is the pth power map. Furthermore there is a lifting of $\gamma_{n}$ to $\Omega S^{2 n+1}\{p\}$.

This, of course, is precisely the fibration sequence $B C M N$ of $\S 1$ with $m=0$. We can now define a delooped version of the spectrum by ${ }^{3}$

$$
V_{(0)}^{n}= \begin{cases}S^{2 k+1}\{p\} & \text { if } n=2 k \\ T^{2 n-1} & \text { if } n=2 k-1\end{cases}
$$

From this we get EHP sequences

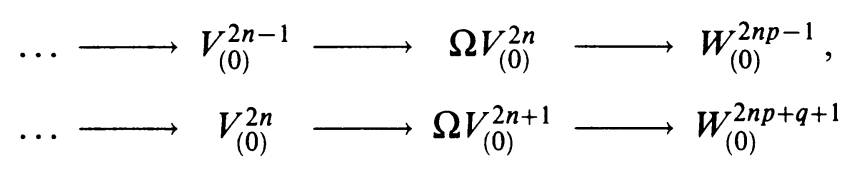

and $\left\{V_{(0)}^{n}\right\} \simeq V(0)$.

Conjecture 3.2. With suitable choices, $W_{(0)}^{2 n p-1} \simeq \Omega V_{(0)}^{2 n p-1}$ (and hence $W_{(0)}^{2 n p+q+1}$ $\simeq \Omega V_{(0)}^{2 n p+q+1}$ ) for $n \geq 1$.

This would be true, for example, if $\phi_{n p} \circ H_{n} \sim *: \Omega^{2} S^{2 n+1} \rightarrow \Omega^{2} S^{2 n p+1} \rightarrow$ $S^{2 n p-1}$. It would give us the most favorable version of the EHP spectrum for $V(0)$.

We now look at the composition question. Given $\beta: S^{k} \rightarrow V_{(0)}^{m}$, we ask for an extension:

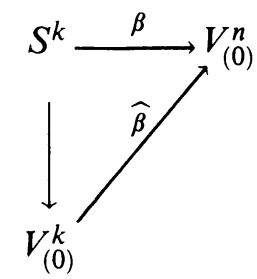

This would enable us to form compositions as in $\S 1$.

We will first examine the implication of such compositions stably. If the multiplication in a ring spectrum can be accomplished by compositions as above, it

\footnotetext{
${ }^{3}$ In [AG], the spaces $V_{(0)}^{n}$ are denoted $T^{n}$, as they form the next spectrum following $S^{n}$. The notation $V_{(0)}^{n}$, although more complicated, is more in keeping with the spirit of this work.
} 
is necessarily homotopy associative. This rules out $V(0)$ when $p=3$ [T2]. On the other hand $V(0)$ is homotopy commutative. To understand the implications of this, let us replace each space $V_{(0)}^{n}$ by the Moore space $P^{n+1}$ which stably carries the homotopy. Suppose that we are given maps $\alpha: S^{k+m-2} \rightarrow P^{m-1}$ and $\beta: S^{l+n-2} \rightarrow P^{n-1}$. We then define extension $\widehat{\alpha}: P^{k+m} \rightarrow P^{m}$ and $\widehat{\beta}: P^{l+n} \rightarrow P^{n}$ of $E \alpha$ and $E \beta$ as the compositions:

$$
\begin{aligned}
P^{k+m} & \simeq P^{2} \wedge S^{k+m-2} \stackrel{1 \wedge \alpha}{\longrightarrow} P^{2} \wedge P^{m-1} \stackrel{\mu}{\longrightarrow} P^{m}, \\
P^{k+l} & \simeq P^{2} \wedge S^{l+n-2} \stackrel{1 \wedge \beta}{\longrightarrow} P^{2} \wedge P^{n-1} \stackrel{\mu}{\longrightarrow} P^{n} .
\end{aligned}
$$

Then if $p>3$, using homotopy associativity of the Moore space spectrum, one can easily see that $\widehat{\alpha}$ and $\widehat{\beta}$ are left module maps over the Moore spectrum, and hence

$$
E^{h}(\widehat{\alpha}) \circ E^{k+m}(\widehat{\beta})=(-1)^{k l} E^{m}(\widehat{\beta}) \circ E^{l+n}(\widehat{\alpha})
$$

as homotopy classes.

The point here is that we use extensions $\widehat{\alpha}$ and $\widehat{\beta}$ constructed from the stable null homotopy of $p$ times the identity of $V(0)$. Unstably, we have

Proposition 3.3. $S^{2 n+1}\{p\}$ has exponent $p$.

Proof. See for example, [CMN1, 1.3].

The question of the exponent of $T^{2 n-1}$ is open. It would suffice to show that $\Omega^{\epsilon} T^{2 n-1}$ has exponent $p$ for some small $\epsilon$. For example, 3.2 and [CMN1, 5.3] implies that $\Omega^{2} T^{2 n p-1}$ has exponent $p$.

Proposition 3.4. Suppose $Y$ is a homotopy commutative homotopy associative $H$ space and $\phi: P^{2 n+1} \rightarrow Y$. Then there is a unique $H$ map $\widehat{\phi}: S^{2 n+1}\{p\} \rightarrow Y$ extending $\phi$.

Lemma 3.5. Suppose that $W X \stackrel{\lrcorner}{\longrightarrow} X \stackrel{\mu}{\longrightarrow} C X$ is an $H$ fibration with a splitting $s: C X \rightarrow X$, and $C X$ is homotopy commutative. Then for each $H$ map $f: X \rightarrow \mathbf{Z}$ with $\mathbf{Z}$ homotopy commutative, the following are equivalent:

(a) $f_{l} \sim *: W X \rightarrow \mathbf{Z}$.

(b) There exists a unique $H$ map $\widehat{f}: C X \rightarrow \mathbf{Z}$ with $\widehat{f} \mu \sim f$.

Proof. Clearly (b) implies (a). Suppose then that $f_{l} \sim *$. Since $\mu(1-s \mu) \sim *$, $1-s \mu \sim \imath \pi$ for some $\pi: X \rightarrow W X$. Now $* \sim f l \pi \sim f-f s \mu$ since $f$ is an $H$ map, so $f \sim f s \mu$. Let $\widehat{f}=f s . \widehat{f}$ is clearly unique. To see that $\widehat{f}$ is an $H$ map consider the diagram:

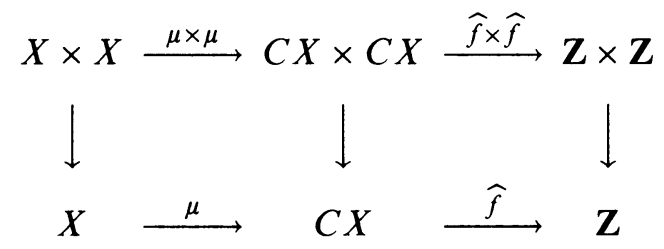

where the commutativity of the right-hand square is in question. However the left-hand square commutes, and the rectangle commutes. Since $\mu \times \mu$ has a right inverse we are done. 
Proof of 3.4. Extend $\phi$ to an $H$ map $\phi_{\infty}: \Omega P^{2 n+2} \rightarrow Y$. We now construct a fibration:

$$
\Omega\left(\bigvee_{n=0}^{\infty} P^{4 n+2 m n+3}\right) \stackrel{g_{2 n+2}}{\longrightarrow} \Omega P^{2 n+2} \stackrel{u_{n}}{\longrightarrow} S^{2 n+1}\{p\}
$$

using [CMN, 11.1]. They construct a homotopy equivalence

$$
\phi_{2 n+2}: S^{2 n+1}\{p\} \times \Omega\left(\bigvee_{n=0}^{\infty} P^{4 n+2 m n+3}\right) \rightarrow \Omega P^{2 n+2}
$$

by multiplying together a map $h_{2 n+2}: S^{2 n+1}\{p\} \rightarrow \Omega P^{2 n+2}$ and a map $g_{2 n+2}$ as above. Thus it suffices to describe $u_{n}$ such that $u_{n} \circ g_{2 n+2} \sim *$ and $u_{n} \circ$ $h_{2 n+2} \sim 1$. Since $S^{2 n+1}\{p\}$ is homotopy associative [N, 4.2] we let $u_{n}$ be the $H$ map extending the inclusion $P^{2 n+1} \subset S^{2 n+1}\{p\}$. Since $S^{2 n+1}\{p\}$ is atomic, $u_{n} \circ h_{2 n+2} \sim 1$. To show that $u_{n} \circ g_{2 n+2} \sim *$, we first note that since it is an $H$ map, it suffices to show that $\left.u_{n} \circ g_{2 n+2}\right|_{P^{2 n+4 n m+2}} \sim *$. However this restriction is a Samelson product and hence the restriction of a commutator. Now by [G6, Proposition 3] (or [N, 4.1]) $S^{2 n+1}\{p\}$ is homotopy commutative, so $u_{n} \circ g_{2 n+2} \sim *$. For the same reason $\phi_{\infty} \circ g_{2 n+2} \sim *$ so by $3.5, \widehat{\phi}$ exists. Given two such extensions $\widehat{\phi}_{1}$ and $\widehat{\phi}_{2}$, they must agree on $\Omega P^{2 n+2}$ since $H$ maps defined on $\Omega P^{2 n+2}$ are determined by their restrictions to $P^{2 n+1}$ up to homotopy. By the uniqueness in 3.5 they are homotopic.

Thus by 3.4 we see that if $Y$ is homotopy commutative and homotopy associative and we are given a null homotopy of $p \cdot I$ in $\Omega^{k} Y$ for $k \leq 2 n$ there is a unique $H$ extension

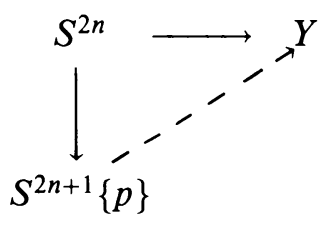

compatible with the null homotopy. We would like to be able to apply this when $Y=S^{2 n+1}\{p\}$ or $Y=T^{2 n-1}$. For the latter, we need to know that $\Omega^{\epsilon} T^{2 n-1}$ has exponent $p$ for some small $\epsilon$, and that $T^{2 n-1}$ is homotopy associative. (Homotopy commutativity follows from [G6, Proposition 3] as before.) Secondly, we would like to construct extensions:

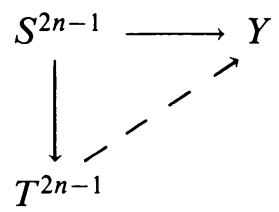

As we shall see, such extensions exist. We have not, however, been able to establish uniqueness. To construct extensions, we need to have more information about the constructions of Anick. In [AG], the authors construct a sequence of cofibrations of co- $H$ spaces

$$
P_{\left(p^{k+1}\right)}^{2 n p^{k}} \stackrel{\alpha_{k}}{\longrightarrow} G_{k-1} \longrightarrow G_{k}
$$


beginning with $G_{0}=P^{2 n+1}$. Here $P_{\left(p^{k+1}\right)}^{2 n p^{k}}$ is the $\mathbf{Z}_{p^{k+1}}$ Moore space of dimension $2 n p^{k}$. The attaching map $\alpha_{k}$ is a co- $H$ map and is divisible by $P^{k}$. Finally, $T^{2 n-1}$ is a retract of $\Omega G_{\infty}$ and $G_{\infty}$ is a retract of $\Sigma T^{2 n-1}$.

Proposition 3.6. Suppose $Y$ is a homotopy commutative homotopy associative $H$ space with exponent $\phi$ and $\phi: P^{2 n} \rightarrow Y$. Then there is an extension:

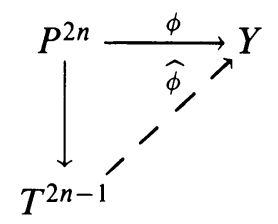

Proof. We first observe from the push out diagram:

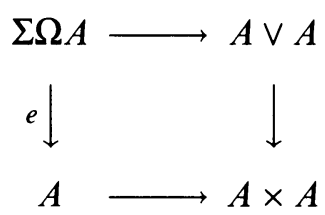

that co- $H$ structures on $A$ correspond to liftings $s: A \rightarrow \Sigma \Omega A$ with $e \cdot s \sim$ 1. Furthermore we claim that co- $H$ maps $\theta: A \rightarrow B$ induce commutative diagrams:

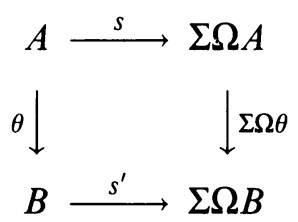

By naturality, it is clear that the diagram commutes when followed by the map $\Sigma \Omega B \rightarrow B \vee B$. However, the inclusion of the fiber of this map, is null homotopic since it is induced from a null homotopic map from $\Omega B$ to $B$.

We now construct, inductively, maps $\phi_{k}: G_{k} \rightarrow \Sigma Y$ with $\phi_{0}=\Sigma \phi$. The inductive step follows from the diagram:

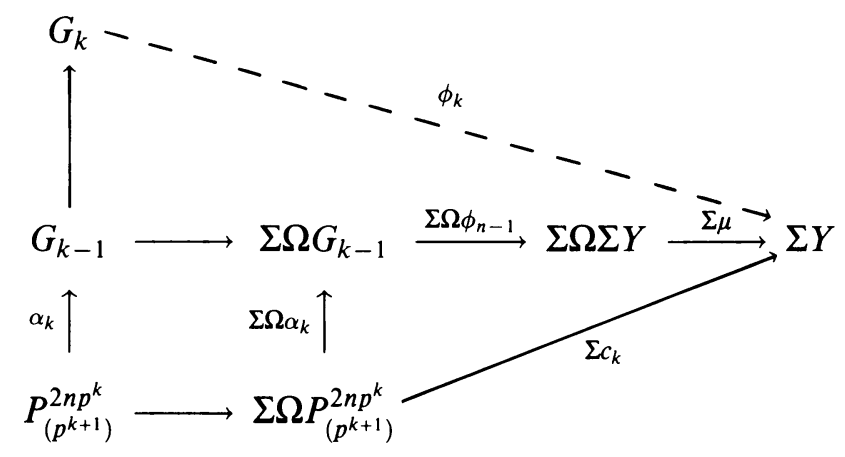

now the composite

$$
P_{\left(p^{k+1}\right)}^{2 n p^{k}} \rightarrow \Sigma \Omega P_{\left(p^{k+1}\right)}^{2 n p^{k}} 2 \stackrel{\Sigma \Omega \alpha_{k}}{\longrightarrow} \Sigma \Omega G_{k-1} \stackrel{\Sigma \Omega \phi_{n-1}}{\longrightarrow} \Sigma \Omega \Sigma Y
$$

is the suspension of $\phi_{n-1} \circ \alpha_{k}$. Hence the entire lower composite is the suspension of $\mu \circ \phi_{n-1} \circ \alpha_{k}$ which is divisible by $p^{k}$. Thus it is trivial and the 
extension exists. This gives a map $\phi_{\infty}: G_{\infty} \rightarrow \Sigma Y$. Now define $\widehat{\phi}$ as the composition

$$
X_{n} \rightarrow \Omega G_{\infty} \stackrel{\Omega \phi_{\infty}}{\longrightarrow} \Omega \Sigma Y \stackrel{\mu}{\longrightarrow} Y
$$

The map $\phi_{\infty}$ can be recovered from $\widehat{\phi}$ as the composite $G_{\infty} \longrightarrow \Sigma X_{(n)} \stackrel{\Sigma \widehat{\phi}}{\longrightarrow} \Sigma Y$; the map $\phi_{\infty}$ is clearly not unique unless it is required to satisfy some stronger property than being an extension of $\phi_{0}=\Sigma \phi$. However given a homotopy of commutativity in $Y$ and a null homotopy of $p$ times the identity in $\Omega^{\epsilon} Y$, the construction of $\widehat{\phi}$ can be made without any choices. It seems, therefore, that there is some hope for a functorial construction.

The universality of $V_{(0)}^{n}$ is reflected in the following freeness property which is easily verified.

Proposition 3.7. $H_{*}\left(V_{(0)}^{n} ; \mathbf{Z}_{p}\right)$ is the free commutative algebra generated by the $\mathbf{Z}_{p}$ module $H_{*}\left(\Sigma^{n} V(0) ; \mathbf{Z}_{p}\right)$.

Proof. This follows immediately from the fibrations:

$$
\begin{aligned}
& \Omega S^{2 n+1} \longrightarrow V_{(0)}^{2 n} \longrightarrow S^{2 n+1} \\
& S^{2 n-1} \longrightarrow V_{(0)}^{2 n-1} \longrightarrow \Omega S^{2 n+1}
\end{aligned}
$$

in which the connecting homomorphism is zero in mod $p$ homology.

Finally, we will use this structure to construct the strong multiplicative structure for $V_{(0)}$ required in $\S 1$; viz.:

$$
V_{(0)}^{n} \wedge V_{(0)}^{m} \longrightarrow V_{(0)}^{m+n}
$$

This translates to maps:

$$
\begin{aligned}
S^{2 n+1}\{p\} \wedge S^{2 m+1}\{p\} & \longrightarrow S^{2 n+2 m+1}\{p\}, \\
S^{2 n+1}\{p\} & \wedge T^{2 m-1} \longrightarrow T^{2 n+2 m-1}, \\
T^{2 n-1} & \wedge T^{2 m-1} \longrightarrow S^{2 n+2 m-1}\{p\} .
\end{aligned}
$$

These are obtained from the adjoints:

$$
\begin{array}{ccc}
S^{2 n+1}\{p\} & \longrightarrow \operatorname{map}_{*}\left(S^{2 m+1}\{p\}, S^{2 n+2 m+1}\{p\}\right), \\
S^{2 n+1}\{p\} & \longrightarrow \operatorname{map}_{*}\left(T^{2 m-1}, T^{2 n+2 m-1}\right), \\
T^{2 n-1} & \longrightarrow \operatorname{map}_{*}\left(T^{2 m-1}, S^{2 n+2 m-1}\{p\}\right)
\end{array}
$$

by using 3.4 and 3.6, assuming in the second case, that $T^{2 n+2 m-1}$ is homotopy commutative. Then these mapping spaces are all homotopy commutative $H$ spaces and the extensions are obtained from the restrictions to the appropriate Moore space. Here we use retractions $\Sigma S^{2 m+1}\{p\} \rightarrow P^{2 m+2}$ and $\Sigma^{2} T^{2 m-1} \rightarrow$ $\Sigma S^{2 m+1}\{p\} \rightarrow P^{2 m+2}$ to construct the restrictions so we need $n \geq 1$ in the first case and $n \geq 2$ in the others.

As in the case $m=-1$, we expect that the third map actually factors through $G_{\infty}^{2 n+2 m-2}$ providing a desuspension:

$$
\Omega\left(T^{2 n-1} \wedge T^{2 m-1}\right) \longrightarrow \Omega G_{\infty}^{2 n+2 m-2} \longrightarrow T^{2 n+2 m-3} .
$$


By the above method it is possible to construct something similar, namely a map

and hence

$$
\Omega T^{2 n-1} \wedge T^{2 m-1} \longrightarrow T^{2 n+2 m-3}
$$

$$
\Omega T^{2 n-1} \wedge \Omega T^{2 m-1} \longrightarrow \Omega T^{2 n+2 m-3} .
$$

4

In this section we will discuss Conjecture 3.2. There are various stronger versions of this conjecture and we will discuss our results to date. It seems to be difficult even to intuit which of the various conjectures will hold up.

We begin with the following:

Conjecture 4.1. There is a map $\Sigma^{2} \Omega^{2} S^{2 n+1} \stackrel{h}{\longrightarrow} P^{2 n p+1}$ which is an epimorphism in $\mathbf{Z}_{p}$ homology.

Proposition 4.2. Conjecture 4.1 implies Conjecture 3.2.

Proof. Since $\Sigma^{2}\left(\Omega^{2} S^{2 n+1}\right) \simeq \Sigma^{2}\left(S^{2 n-1} \times B W_{n}\right)$ by [G4], 4.1 is equivalent to having a map $h^{\prime}: \Sigma^{2} B W_{n} \rightarrow P^{2 n p+1}$ which is a $\mathbf{Z}_{p}$ homology epimorphism. Now $P^{2 n p}$ is the $2 n p$ skeleton of $T^{2 n p-1}$. Since $T^{2 n p-1}$ is an $H$ space, there is an extension $\Omega P^{2 n p+1} \rightarrow T^{2 n p-1}$. Thus the adjoint of $h^{\prime}$ gives a map $B W_{n} \stackrel{h^{\prime}}{\longrightarrow} \Omega^{2} P^{2 n p+1} \rightarrow \Omega T^{2 n p-1}$. Both spaces have isomorphic $\mathbf{Z}_{p}$ cohomology which is a tensor product of divided polynomial algebras and exterior algebras. All of the generators are tied together by the operations $\beta$ and $\mathscr{P}^{1}$ in such a way that a map inducing an isomorphism in dimension $2 n p-2$ induces an isomorphism in all cohomology groups (as in the atomicity proof in [CM1]). Thus $B W_{n} \simeq \Omega T^{2 n p-1}$. Since $V_{0}^{2 n p-1}=$ fiber of $\varphi_{n}=B W_{n}$, we are done.

We will hazard a further conjecture that may seem to some to be uncautious. This concerns the number of suspensions necessary to split $\Omega^{2} S^{2 n+1}$ when $S^{2 n+1}$ is localized at an odd prime. At one time Barratt [B] conjectured that the Snaith splitting [S]:

$$
\Sigma^{\infty}\left(\Omega^{n} \Sigma^{n} X\right) \simeq \Sigma^{\infty}\left(\bigvee_{k=0}^{\infty} D_{k}(X)\right)
$$

could be obtained after $n$ suspensions. It was later proven by Kirley [K] that the maps in the Snaith splitting could not be desuspended to split $\Omega^{n} \Sigma^{n} X$ after a finite number of suspensions if $H_{*}\left(X ; \mathbf{Z}_{2}\right) \neq 0$. Cohen $[C]$ also proved that one needs $2^{n}$ suspensions to split off $D_{2^{n-1}}(X)$ when $D_{2^{n-1}}(X)$ is not contractible. Mahowald $[\mathrm{M}]$ has a particularly nice description of the problem when $X=S^{2 n-1}$ localized at 2: The pieces $D_{2^{k-1}}$ and $D_{2^{k}}$ hold onto each other until the Freudenthal suspension theorem demands that they split. None of these results applies to $\Omega^{2} S^{2 n+1}$ localized at an odd prime. In particular, Mahowald's graphic description of the problem does not have an analogy away from 2.

Conjecture 4.3. $\Sigma^{2} \Omega^{2} S^{2 n+1} \simeq \Sigma^{2}\left(\bigvee_{k \geq 0} D_{k}\left(S^{2 n-1}\right)\right)$ when localized at an odd prime. Clearly 4.3 implies 4.1 .

We observe that using the techniques of [CG, Lemma 2.3] it suffices to construct maps $\Sigma^{2} D_{p^{i}}\left(S^{2 n-1}\right) \stackrel{\alpha_{i}}{\longrightarrow} \Sigma^{2} \Omega^{2} S^{2 n+1}$ for each $i \geq 1$. Furthermore, having 
constructed $\alpha_{1}, \ldots, \alpha_{n}$ it follows that the splitting 4.3 works up through the $(2 n p-2) p^{n+1}-1$ skeleton. Now $\alpha_{1}$ clearly exists.

Proposition 4.4. There is a map $\Sigma^{2} D_{p^{2}}\left(S^{2 n-1}\right) \stackrel{\alpha_{2}}{\longrightarrow} \Sigma^{2} \Omega^{2} S^{2 n+1}$ inducing a monomorphism in $\mathbf{Z}_{p}$ homology.

Proof. We first observe that we may replace $\Omega^{2} S^{2 n+1}$ with $B W_{n}$ since

$$
\Sigma^{2}\left(\Omega^{2} S^{2 n+1}\right) \simeq \Sigma^{2}\left(S^{2 n-1} \times B W_{n}\right)
$$

(see [G4] or [G5]). Next we use the action of a $p$ local unit $k: S^{2 n-1} \rightarrow S^{2 n-1}$ to induce a map $[k]: B W_{n} \rightarrow B W_{n}$. Forming telescopes in the usual manner we can split $\Sigma B W_{n}$ :

$$
\Sigma B W_{n} \simeq X_{1} \vee \cdots \vee X_{p-1}
$$

where $H_{*}\left(X_{i} ; \mathbf{Z}_{p}\right)$ contains all monomials of $H_{*}\left(B W_{n} ; \mathbf{Z}_{p}\right)$ whose length is congruent to $i \bmod (p-1)$. Thus the $2 n p^{2}$ skeleton of $X_{1}$ is

$$
S^{2 n p-1} \cup_{p l} e^{2 n p} \cup e^{(2 n p-2) p+1} \cup_{p l} e^{(2 n p-2) p+2} \cup e^{2 n p^{2}-1} \cup_{p l} e^{2 n p^{2}} .
$$

There is a map $S^{2 n p-1}\{p\} \rightarrow B W_{n}$ inducing a monomorphism in $\mathbf{Z}_{p}$ homology. Since $\Sigma S^{2 n p-1}\{p\}$ is a wedge of Moore spaces [G1], the $2 n p^{2}$ skeleton of $X_{1}$ is

$$
\left(P^{2 n p} \vee P^{(2 n p-2) p+2}\right) \cup_{\theta} e^{2 n p^{2}-1} \cup_{p l} e^{2 n p^{2}} .
$$

Now we assert that the suspension of the composite:

$$
\theta_{1}: p^{2 n p^{2}-1} \underset{\theta}{\longrightarrow} P^{2 n p} \vee P^{(2 n p-2) p+2} \rightarrow P^{2 n p}
$$

is null homotopic. Hence $\left(\Sigma X_{1}\right)^{2 n p^{2}+1} \simeq P^{2 n p+1} \vee \Sigma^{2} D_{p^{2}}\left(S^{2 n-1}\right)$ and the proof is complete.

To prove this we first observe that the composite

$$
P^{2 n p+1} \subset \Sigma^{1} X_{1} \rightarrow \Sigma^{2} B W_{n} \rightarrow \Sigma^{2} \Omega^{2} S^{2 n p+1} \rightarrow S^{2 n p+1}
$$

induces an epimorphism in $\mathbf{Z}_{p}$ homology. It follows that the composite:

$$
P^{2 n p^{2}} \stackrel{E \theta_{1}}{\longrightarrow} P^{2 n p+1} \longrightarrow S^{2 n p+1}
$$

is null homotopic. We now introduce the space $\left\{T_{2 n p+1}\right\}$ from [CMN3, Theorem 3] and the fibration sequence which appears vertically in the diagram:

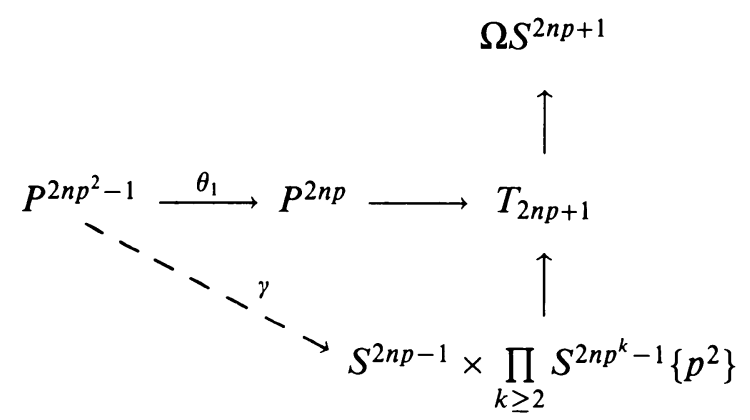

where the above null homotopy defines $\gamma$. There are only two possibly non nullhomotopic components of $\gamma$. Consider first $\gamma_{2}: P^{2 n p^{2}-1} \rightarrow S^{2 n p^{2}-1}\left\{p^{2}\right\}$. Such maps are detected in homology and the map $S^{2 n p^{2}-1}\left\{p^{2}\right\} \rightarrow T_{2 n p+1}$ induces a 
monomorphism in $\mathbf{Z}_{p}$ homology. Since $\left(\theta_{1}\right)_{*}=0$ we conclude that $\gamma_{2}=0$. Thus $\gamma=\gamma_{1}: P^{2 n p^{2}-1} \rightarrow S^{2 n p-1}$. Now the adjoint of the composite

$$
P^{2 n p^{2}-1} \stackrel{\theta_{1}}{\longrightarrow} P^{2 n p} \longrightarrow T_{2 n p+1} \longrightarrow \Omega P^{2 n p+1}
$$

is $E \theta_{1}$ which is stably trivial, so $\gamma_{1}$ is stably divisible by $p$. This is impossible for maps defined on $P^{2 n p^{2}-1}$, so $\gamma_{1}$ is stably trivial. But $\left[P^{2 n p^{2}-1}, S^{2 n p-1}\right] \cong$ $\left\{P^{2 n p^{2}-1}, S^{2 n p-1}\right\}$ so $\gamma_{1} \sim *$ and hence $E \theta_{1} \sim *$.

One of the important tools in EHP calculations is the map $B \Sigma_{p} \rightarrow Q\left(S^{0}\right)$. Let $B$ be the localization of $B \Sigma_{p}$ at $p$. Then we have restrictions:

$$
\begin{gathered}
B^{n q} \stackrel{\lambda}{\longrightarrow} \Omega^{2 n+1} S^{2 n+1}, \\
B^{n q-1} \stackrel{\lambda}{\longrightarrow} \Omega^{2 n} \widehat{S}^{2 n}
\end{gathered}
$$

and the cofibration sequence of $B^{n q}$ maps to the EHP fibration sequences:

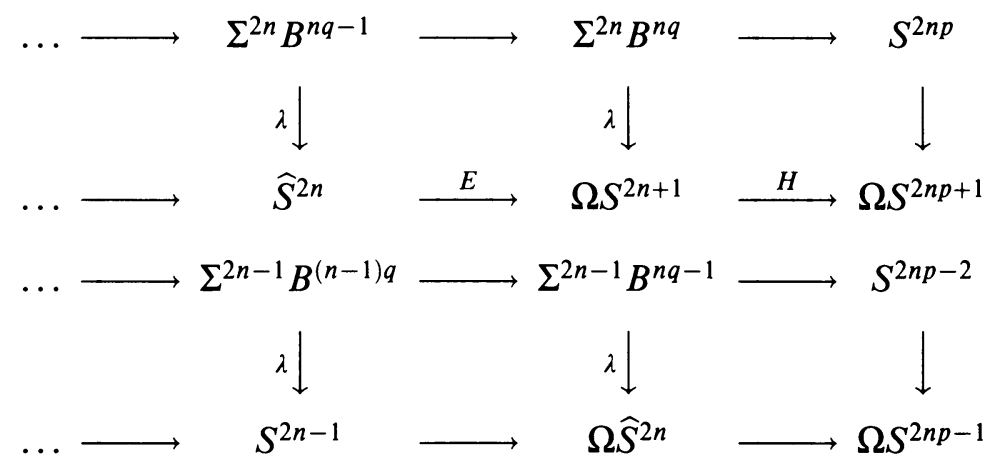

(See [G2, Proposition 11].) It would be desirable to have similar constructions in other cases. In fact such a construction for $V(0)$ can easily be made (after Ravenel $[R])$. Consider the diagram of cofibrations defining the right-hand terms:

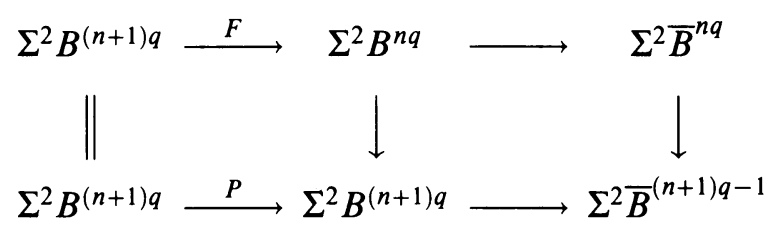

where $F$ is the unique compression of $P=p l \wedge 1$. (See [G2, Proposition 7].) Note that $\Sigma^{2} \bar{B}^{n q}$ may not be a suspension. By a slight variant of Lemma 21 of [G2] we get a commutative diagram:

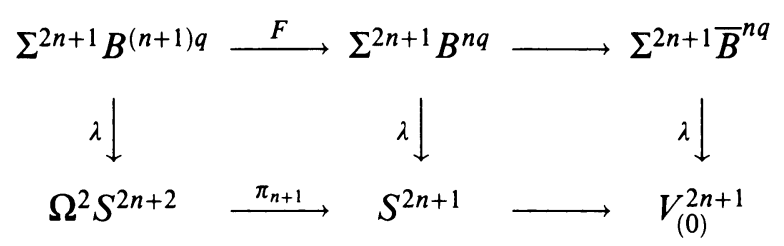


More easily one gets

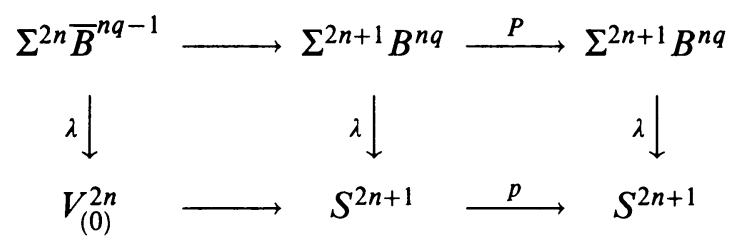

This gives the EHP ladders:

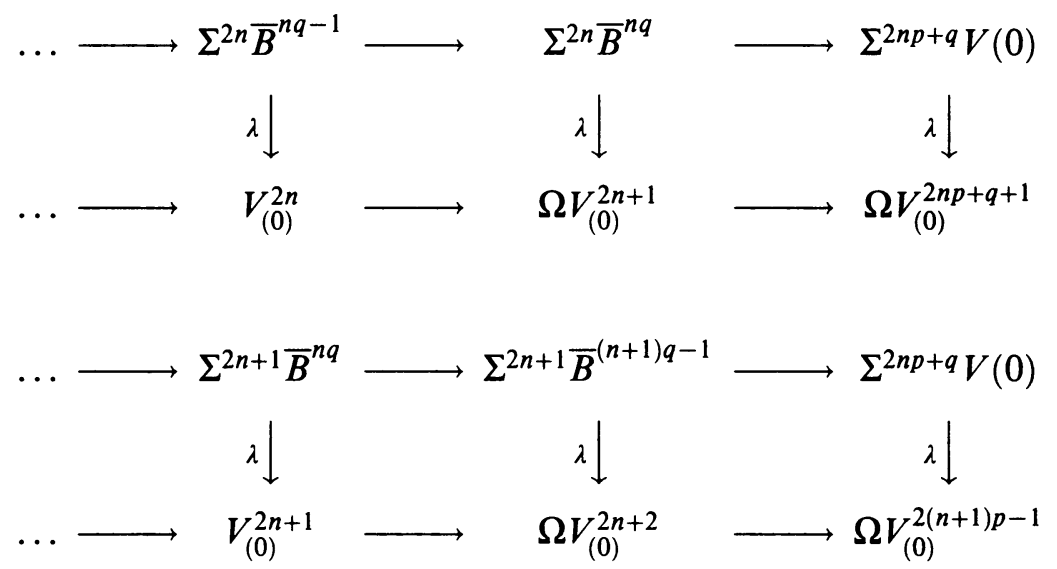

since $\bar{B}^{n q} / \bar{B}^{n q-1}=\Sigma^{(n+1) q} V(0)$ and $\bar{B}^{(n+1) q-1} / \bar{B}^{n q}=\Sigma^{(n+1) q-1} V(0)$. We wonder whether there are also Snaith maps in this context.

One consequence is the commutative diagram:

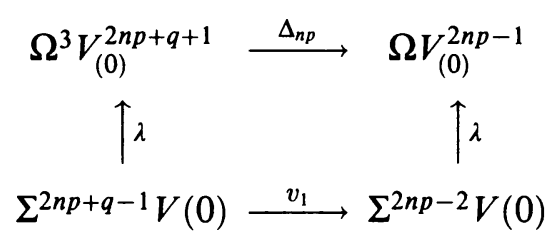

Thus if $V_{(0)}$ satisfies $\mathrm{CMN}$, the maps $\pi_{n}$ represent $v_{1}$ on the bottom Moore space and the spectrum $\left\{V_{(1)}^{n}\right\}$ is stably equivalent to $V(1)$.

If $p \geq 5$ Ravenel goes on. He constructs a map $\Sigma^{2 p} \bar{B}^{(n+p) q} \longrightarrow \Sigma^{2} \bar{B}^{n q}$ which is a compression of $v_{1}: \Sigma^{2 p} \bar{B}^{(n+p) q} \longrightarrow \Sigma^{2} \bar{B}^{(n+p) q}$ and represents $v_{2}$. In fact there is an obstruction theory to this process in general. We seek finite spectra $B_{(m)}^{n q}$ and maps $h_{m}: B_{(m)}^{n q} \longrightarrow B^{n q+q_{m+1}} \wedge V(m)$ inducing a monomorphism in $\mathrm{Z}_{p}$ homology. Here $B_{(-1)}^{n q}=B^{n q}$ and $B_{(0)}^{n q}=\bar{B}^{n q}$. Inductively one seeks a 
lifting $f_{m-1}$ in the diagram:

$$
\begin{aligned}
& \Sigma^{q_{m}} B_{(m-1)}^{\left(n+p^{m}\right) q} \quad \stackrel{\Sigma^{q_{m}} h_{m-1}}{\longrightarrow} \quad B^{n q+q_{m+1}} \wedge \Sigma^{q_{m}} V(m-1) \\
& f_{m-1} \downarrow \quad 1 \wedge v_{m} \downarrow \\
& B^{n q+q_{m-1}} \longrightarrow B_{(m-1)}^{\left(n+p^{m}\right) q} \stackrel{h_{m-1}}{\longrightarrow} \quad B^{n q+q_{m+1}} \wedge V(m-1) \\
& \downarrow \\
& B_{(m)}^{n q} \quad \stackrel{h_{m}}{\longrightarrow} \quad B^{n q+q_{m+1}} \wedge V(m)
\end{aligned}
$$

This actually works when $m=0,1$. One can then calculate $Q^{m+1}\left(a_{k} \wedge u\right)=$ $b_{k+p^{m}+\cdots+1} \wedge u$ where $a_{k} \in \widetilde{H}^{k q-1}\left(B ; \mathbf{Z}_{p}\right), b_{k}=\beta a_{k}$ and $u \in \widetilde{H}^{\circ}\left(V(m) ; \mathbf{Z}_{p}\right)$ are the respective generators. These classes are nonzero in $B_{(m)}^{n q}$ (in fact in $\left.B_{(-1)}^{n q}\right)$ for $n$ large and one obtains that $B_{(m)}^{n q} / B_{(m)}^{k q}$ is filtered by $n-k$ copies of $V(m+1)$. Compatible maps $\Sigma^{2 n+1} B_{(m)}^{n q} \rightarrow V_{(m)}^{2 n+1}$ allow this information to be transferred and we conclude that the maps $\pi_{n p}: \Omega^{2} V_{(m)}^{2 n p+q_{m+1}+1} \rightarrow V_{(m)}^{2 n p-1}$ induce $v_{m+1}$. Thus, inductively we produce the spectra $V(m+1)$.

\section{6}

In this section we will consider the problem of constructing Cohen-MooreNeisendorfer maps for the spectrum $V(0)$. There is a clear procedure which imitates the analysis of [CNM4]. In order for it to work most efficiently we will assume that $V_{(0)}^{2 n-1}=T^{2 n-1}$ is homotopy associative (and hence homotopy commutative).

What we seek, then, is the condition CMN for $V_{(0)}$ namely, maps

so that the diagrams:

$$
\pi_{n}: \Omega^{2} V_{(0)}^{2 n+q+1} \rightarrow V_{(0)}^{2 n-1}
$$

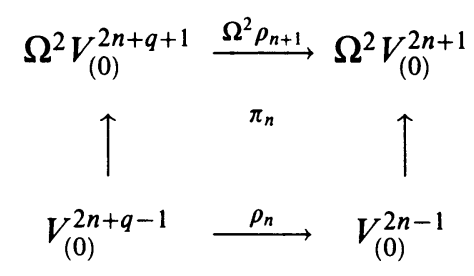

Here $\rho_{n}$ will be given by extending the Adams map:

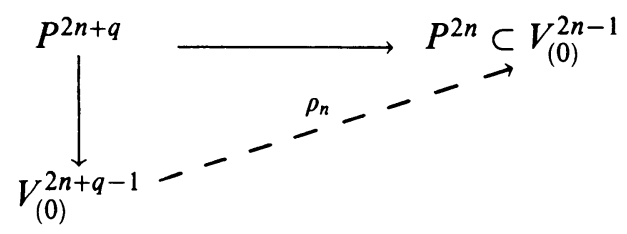

using 13.6. 
Now let $\Sigma^{2 n} V(1)=P^{2 n+1} \cup_{v_{1}} C P^{2 n+2 p-1}$ with $n \geq 2$. Let $\mu_{n}$ be the composite:

$$
\Sigma^{2 n} V(1) \rightarrow P^{2 n+2 p} \rightarrow V_{(0)}^{2 n+q+1} .
$$

Since $\rho_{n} \mu_{n} \sim *$, we get a commutative diagram of fibrations:

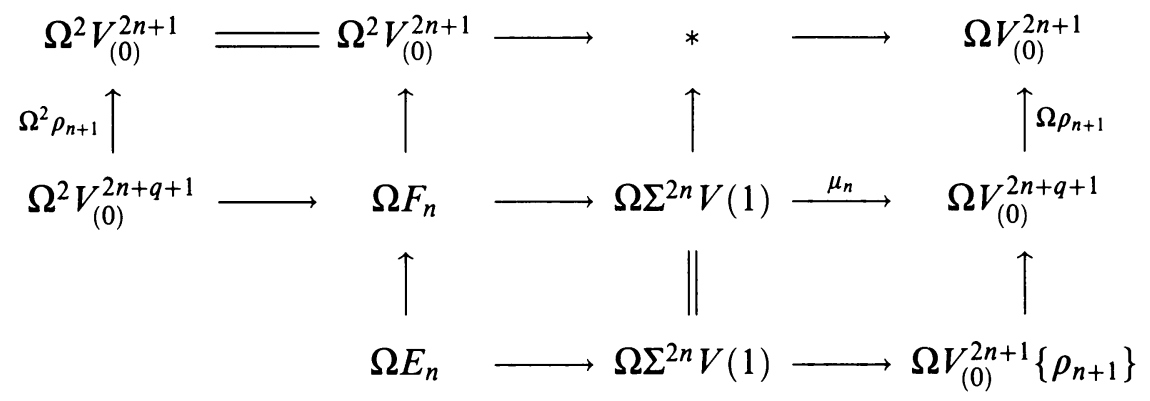

Problem 6.1. Decompose $\Omega F_{n}$.

The most favorable outcome would produce a map $\theta: \Omega F_{n} \rightarrow V_{(0)}^{2 n-1}$. We would then write $\pi_{n}: \Omega^{2} V_{(0)}^{2 n+q+1} \rightarrow \Omega F_{n} \rightarrow V_{(0)}^{2 n-1}$ where $\Omega^{2} \rho_{n+1} \simeq E^{2} \circ \pi_{n}$ and $\left.\pi_{n}\right|_{V_{(0)}^{2 n+q-1}} \simeq \rho_{n}$ by uniqueness. This, of course, is a direct analog of [CMN4]. One would hope to use the same techniques as far as possible. If $p>5$, $\pi_{*}(\quad ; V(1))$ is a differential Lie algebra and one can construct Samelson products in $H_{*}\left(\Omega F_{n}\right)$. The situation is not entirely analogous, however. $\mu_{n}$ actually factors through $V_{(0)}^{2 n+q}$, creating a new fiber $\widetilde{F}_{n}$ which is more analogous to the previous case. There is much work to be done on this problem.

\section{APPENDIX}

Extensive calculations have been done in the case $m=0,1,2$ and $\infty$ at the prime 3. Assuming a Barrat-Toda formula and occasionally the maps of $\S 5$, we have been able to calculate the EHP sequences, even though they may not correspond to the homotopy of spaces. They do, however give $\pi_{*}(V(m))$ with the exception that, for $p=3$, we limit our range of dimensions. The nonassociativity of $\pi_{*}(V(0))$ appears in homotopy in dimension 49. The following table shows $v_{1}$ torsion part of the spectral sequence for $p=5$. Note the reluctance of differential to cross certain filtrations. The pieces that hold together correspond to copies of $A_{1}$ made from $p$ copies of $V(1)$. 
EMP SPECTRA AND PERIODICITY. I: GEOMETRIC CONSTRUCTIONS

TABle. $v_{1}$ torsion in $\kappa_{*}(V(0))$ for $p=5$

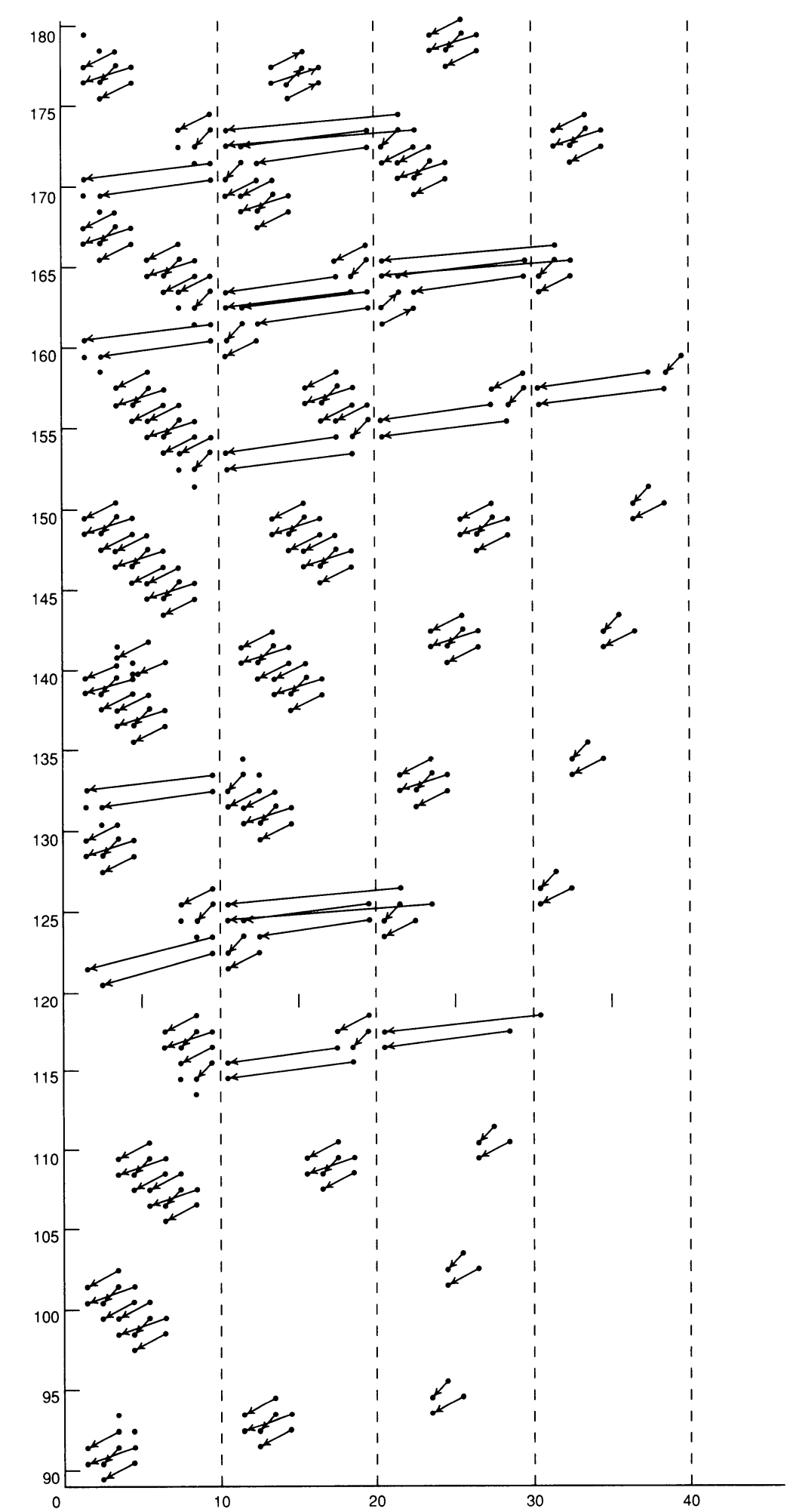




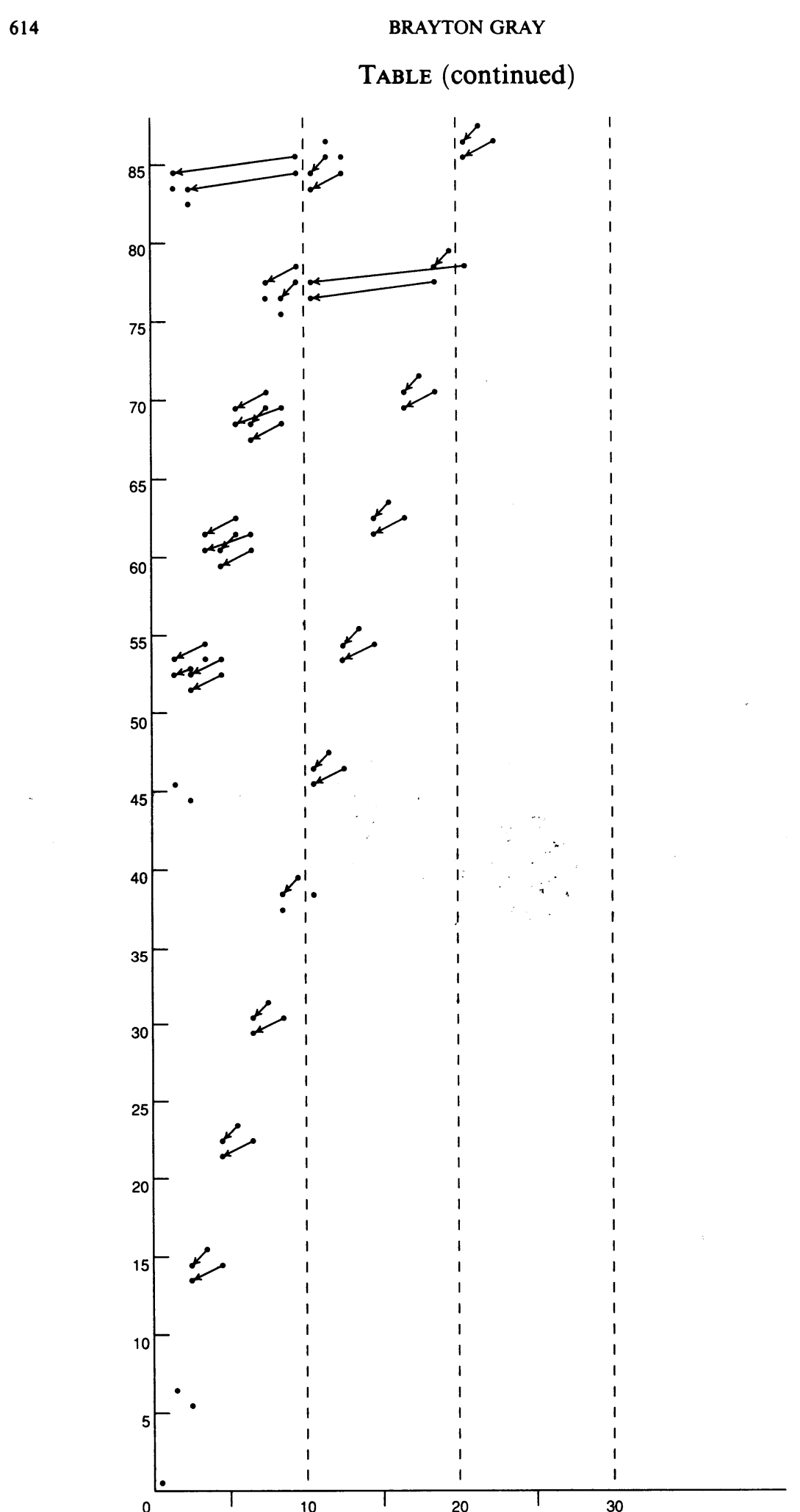




\section{REFERENCES}

[A] D. Anick, Differential algebras in topology, Lecture Notes in Math., A. K. Peters Ltd., 1993.

[AG] D. Anick and B. Gray, Small $H$ spaces related to Moore spaces, Topology (submitted).

[B1] M. G. Barratt, Note on a formula due to Toda, J. London Math. Soc. 36 (1961), 95-96.

[B2] _ private communication.

[BC] A. K. Bousfield and E. B. Curtis, A spectral sequence for the homotopy of nice spaces, Trans. Amer. Math. Soc. 151 (1970), 457-479.

[BH] M. G. Barratt and P. J. Hilton, On joint operations of homotopy groups, Proc. London Math. Soc. 3 (1953), 430-445.

[CG] R. L. Cohen and P. Goerss, Secondary cohomology operations that detect homotopy classes, Topology 23 (1984), 177-194.

[CM1] F. R. Cohen and M. E. Mahowald, Unstable properties of $\Omega^{n} S^{n+k}$, Contemp. Math., vol. 12, Amer. Math. Soc., Providence, R.I., 1982, pp. 81-90.

[CM2] — The splitting of $\Omega^{2} S^{2 n+1}$, Algebraic Topology (Seattle, 1985), Lecture Notes in Math., vol. 1286, Springer-Verlag 1987, pp. 188-192.

[CMN1] F. R. Cohen, J. C. Moore, and J. A. Neisendorfer, Torsion in homotopy groups, Ann. of Math. 109 (1979), 121-168.

[CMN2] _ The double suspension and exponents of the homotopy groups of spheres, Ann. of Math. 110 (1979), 549-565.

[CMN3] _ Exponents in homotopy theory, Algebraic Topology and Algebraic $K$ Theory, Ann. of Math. Stud., no. 113, Princeton Univ. Press, 1987, pp. 3-34.

[CMN4] _ Decomposition of loop spaces and applications to exponents, Algebraic Topology (Aarhus, 1978), Lecture Notes in Math., vol. 763, Springer-Verlag, 1979, pp. 1-12.

[G1] B. Gray, On the homotopy groups of mapping cones, Proc. London Math. Soc. (3) 26 (1973), 497-520.

[G2] _ Unstable Families related to the image of $J$, Math. Proc. Cambridge Philos. Soc. 96 (1984), 95-113.

[G3] —, On Toda's fibrations, Math. Proc. Cambridge Philos. Soc. 97 (1985), 289-298.

[G4] _ On the double suspension, Proc. of the Arcata Conference, 1986, Lecture Notes in Math., vol. 1370, Springer-Verlag, pp. 150-162.

[G5] _ On the iterated suspension, Topology 27 (1988), 301-310.

[G6] _- Homotopy commutativity and the EHP sequence, Proc. Internat. Conf., 1988, Contemp. Math., vol. 96, Amer. Math. Soc., Providence, R.I., 1989, pp. 181-188.

[G7] _ EHP spectra and periodicity. II: $\Lambda$ algebra models, Trans. Amer. Math. Soc.

[H1] J. R. Harper, A proof of Gray's conjecture, Proc. Internat. Conf., 1988, Contemp. Math., vol. 96, Amer. Math. Soc., Providence, R. I., 1989, pp. 181-188.

[K] P. O. Kirley, On the indecomposability of iterated loop spaces, Ph.D. Thesis, Northwestern Univ., 1975.

[N] J. A. Neisendorfer, Properties of certain $H$ spaces, Quart. J. Math. Oxford Ser. 34 (1983), 201-209.

[R] D. C. Ravenel, private communication.

[Sm] L. Smith, On realizing complex carbodism modules IV, Applications to the stable homotopy groups of spheres, Amer. J. Math. 99 (1971), 418-436.

[Sn] V. Snaith, A stable decomposition of $\Omega^{2} S^{n} X$, J. London Math. Soc. (2) 7 (1974), 577-583.

[T1] H. Toda, Composition methods in homotopy groups of spheres, Princeton Univ. Press, 1962. 
[T2] _ An important relation in homotopy groups of spheres, Proc. Japan Acad. 43 (1967), 839-842.

[T3] __ On spectra realizing certain exterior parts of the Steenrod algebra, Topology 10 (1971), 53-65.

Department of Mathematics, University of Illinois at Chicago, Chicago, Illinois 60680

Department of Mathematics, Northwestern University, Evanston, Illinois 60208

E-mail address: brayton@math.neru.edu 